\title{
Effect of Oxygen Redistribution on Temperature Dependence of Pseudo-Gap in Underdoped $\mathrm{HoBa}_{2} \mathrm{Cu}_{3} \mathrm{O}_{7-\delta}$ Single Crystals
}

\author{
R.V. Vovk ${ }^{a}$, Z.F. NAzyrov ${ }^{a}$, M.A. Obolenskit ${ }^{a}$, V.M. Pinto Simoes ${ }^{b}$, M. JanuszczyK $^{c}$ \\ AND J.N. LATOSIŃSKA ${ }^{c, *}$ \\ ${ }^{a}$ Kharkov National University, 4 Svoboda Sq., 61077 Kharkov, Ukraine \\ ${ }^{b}$ IPA_Instituto Superior Autónomo de Estudos Politécnicos, Rua de Xabregas, 20, $1^{\circ}$ 1900-440 Lisboa, Portugal \\ ${ }^{c}$ Institute of Physics, Adam Mickiewicz University, Umultowska 85, 61-614 Poznań, Poland
}

(Received January 7, 2011; in final form March 20, 2011)

\begin{abstract}
The effect of annealing at room temperature on the excess conductivity of oxygen deficient $\mathrm{HoBa}_{2} \mathrm{Cu}_{3} \mathrm{O}_{7-\delta}$ single crystal is studied. It is shown that there is substantial extension of the interval of linear dependence $\rho_{a b}(T)$ and narrowing of the temperature area of the pseudo-gap regime as a result of annealing. The excess conductivity satisfies the exponential temperature dependence in a wide temperature interval. The temperature dependence of the pseudogap is satisfactorily described by the BCS-BEC theoretical model.
\end{abstract}

PACS: 74.72.-h

\section{Introduction}

Pseudogap anomaly (PA) in high temperature superconductor (HTS) compounds is an important problem that has attracted great interest of researchers [1-3]. According to modern concepts, this phenomenon could be a clue to understanding the nature of HTS $[2,3]$. Electrical measurements of the transition to pseudogap (PG) regime suggests a faster than linear decline in the value of electroresistance in basic planes, $\rho_{a b}(T)$ (the appearance of the so-called excess conductivity), when temperature falls below the characteristic value $T^{*}[1,3]$. Compounds of the formula $\mathrm{YBa}_{2} \mathrm{Cu}_{3} \mathrm{O}_{7-\delta}$ (also known as $\mathrm{YBCO}$ or 1-2-3 system) and their rare-earth analogues are particularly interesting for the study of PG anomaly. The interest in YBCO systems has been prompted by the progress in the methods of single crystals investigation [1], strong dependence of conductivity and critical parameters of these systems from the oxygen stoichiometry and the possibility of replacement of yttrium (Y) with its iso-analogues. When yttrium is replaced by holmium (Ho), which has a magnetic moment higher than $10 \mu_{\mathrm{B}}$, the resulting $\mathrm{HoBa}_{2} \mathrm{Cu}_{3} \mathrm{O}_{7-\delta}$ compound is paramagnetic in the ground state. Importantly, the substitution of $\mathrm{Y}$ with Ho in the optimum oxygen doped samples does not affect substantially the superconducting properties [4] thanks to the fact that Ho is located away from the superconducting planes, which in turn interfere with the formation of long-range magnetic order. More interesting for inves-

\footnotetext{
* corresponding author; e-mail: Jolanta.Latosinska@amu.edu.pl
}

tigation are oxygen underdoped samples [4]. In these compounds the rare earth ion is sensitive to the local symmetry of its environment and to the charge density distribution. Both factors affect the crystal field responsible for the electronic structure of the rare-earth ion.

An important peculiarity of the 1-2-3 system is the ability to realize a non-equilibrium state under a specific oxygen non-stoichiometry $[5,6]$, which may be initiated by external factors such as temperature [5] or high pressure [6]. This non-equilibrium state is accompanied by a redistribution process of the labile oxygen and structural relaxation, which in turn affect substantially the electro-transport parameters of the system $[5,6]$ and, in particular the temperature dependence of electric resistance. To the best of our knowledge, experimental studies of labile oxygen redistribution in non-equilibrium state (at fixed oxygen concentration in the sample) on PS-anomaly in the system of 1-2-3 have not been so far reported in literature. Thus in the present study we investigate the effect of maintenance of the $\mathrm{HoBa}_{2} \mathrm{Cu}_{3} \mathrm{O}_{7-\delta}$ single crystal at room temperature on its excess conductivity after it has been annealed to induce oxygen deficiency.

\section{Material and method}

The single crystals of $\mathrm{HoBa}_{2} \mathrm{Cu}_{3} \mathrm{O}_{7-\delta}$ were grown using the solution-melt technique in a gold crucible described in the previous works [1,4]. Electric contacts were made according to the standard four-contact scheme by applying silver paste onto the crystal surface and on the contact surface of silver conductors (of $0.05 \mathrm{~mm}$ in diameter). 
The crystals were annealed at a temperature of $200^{\circ} \mathrm{C}$ in an oxygen atmosphere for three hours to reduce the resistance in between the contacts to less than $1 \Omega$ and to allow measurements with a current of $10 \mathrm{~mA}$ in the $a b$-plane. The measurements were carried out in the temperature drift mode using the two opposite directions of the transport current so as to eliminate the impact of parasitic signal. The temperature was measured using a platinum thermoresistor; the voltage was measured across the sample by the reference resistor with V2-38 nanovoltmeters. As the critical temperature $\left(T_{\mathrm{c}}\right)$ of superconductive transition we accepted the one corresponding to the main maximum in the $\mathrm{d} \rho_{a b}(T) / \mathrm{d} T$ dependence.

To decrease the oxygen concentration, the crystal was annealed in the air flow, at a temperature of $650{ }^{\circ} \mathrm{C}$ for $24 \mathrm{~h}$. After annealing, the crystal was cooled for 2-3 min until it reached room temperature. It was cooled up to the liquid nitrogen temperature within 10-15 min and then to the liquid helium temperature. All the measurements of electric resistance were performed for the annealed sample. To study the influence of the annealed sample maintenance at room temperature, after the first $\rho(T)$ measurement, we kept the sample at room temperature for a few hours and only then we repeated the measurement. We repeated the procedure several times. The last series of measurements were taken after an additional maintenance of the sample at room temperature for 5 days.

\section{Results and discussion}

The temperature dependence of the excess conductivity $\mathrm{HoBa}_{2} \mathrm{Cu}_{3} \mathrm{O}_{7-\delta}$ single crystals was measured just after inducing the oxygen deficiency (curve 1) and then after the step by step cooling of the annealed sample to room temperature at which it was kept for $20 \mathrm{~h}$ (curve 2) and 5 days (curve 3 ) without changing the oxygen content within the volume of the crystal, Fig. 1.

The inset (a) of Fig. 1 shows the temperature dependence of the resistivity of the crystal in the basis $a b$-plane, $\rho_{a b}(T)$ (parts of the curves in Fig. 1 are not shown for clarity). The increase in the annealing time caused an increase in the critical temperature $\left(T_{\mathrm{c}}\right)$ of the sample, a decrease in its the electroresistance and reduction of the linear area of the $\rho_{a b}(T)$ dependence, which in the high temperature range was substantially extended. The last observation is reflected by the decrease in the characteristic value $T^{*}$ at which the curves start to systematically deviate from linearity. According to previous studies, $T^{*}$ is the temperature at which the $\mathrm{PG}$ regime begins [1-4]. As is known (see, for example [2]) depending on the disorder strength and the hole concentration within the conducting $\mathrm{Cu}-\mathrm{O}$ planes this deviation may be positive (upturn) or negative (downturn). Apparently from Fig. 1, the deviation is negative because for carrying out of our experiment, samples of such structure that the deviation occurred strictly downwards have been prepared.

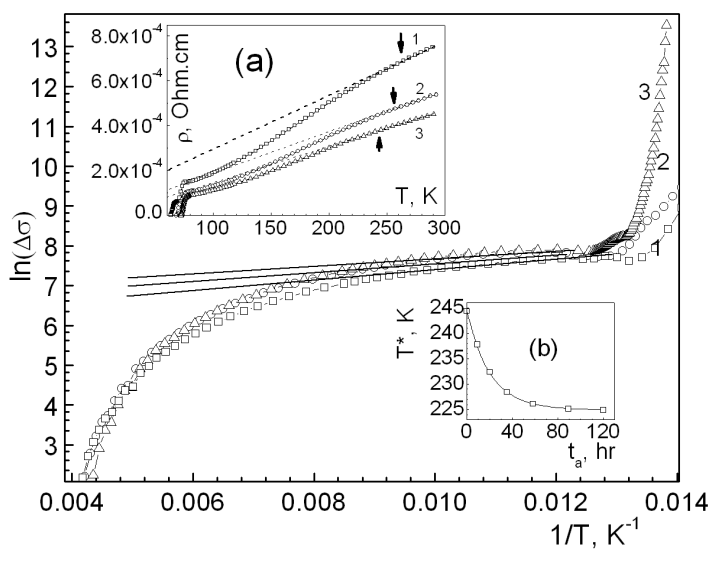

Fig. 1. Temperature dependence of excess conductivity $\mathrm{HoBa}_{2} \mathrm{Cu}_{3} \mathrm{O}_{7-\delta}$ single crystals in $\ln \Delta \sigma$ versus $1 / T$ coordinates, measured after inducing oxygen deficiency by annealing (curve 1) and after the step by step cooling the sample to room temperature at which it was kept for $20 \mathrm{~h}$ (curve 2) and 5 days (curve 3) without changing the oxygen content within the volume of the crystal. Inset (a): temperature dependence of resistivity in the basis plane $\rho_{a b}(T)$. The arrows show the temperatures at which the systematic deviation of the experimental curves from linearity starts. Inset (b): the time dependence of temperature $T^{*}$ upon the gradual cooling of the annealed samples to room temperature $T^{*}(t)$. Solid lines represent the fit of the experimental data with Eq. (7).

In this area of the phase diagram Babayev and Kleinert theoretical model [7] (see below) to which we compare our experimental data is applicable.

The decrease in the $\rho_{a b}(T)$ value, observed at temperatures $T<T^{*}$, indicates the appearance of the so-called excess conductivity $\Delta \sigma$ in the crystal. The temperature dependence of the excess conductivity is usually determined from the equation

$$
\Delta \sigma=\sigma-\sigma_{0},
$$

where $\sigma_{0}=\rho_{0}^{-1}=(A+B T)^{-1}$ is the conductivity obtained from the extrapolation of the linear part to the zero temperature, and $\sigma=\rho^{-1}$ is the experimental value of the conductivity in the ground state. The $\Delta \sigma(T)$ dependence is shown in Fig. 1 in the $\ln \Delta \sigma$ versus $1 / T$ coordinates. It is observed that in a wide temperature interval all experimental curves are linear, in agreement with the analytical expression

$$
\Delta \sigma \sim \exp \left(\Delta_{a b}^{*} / T\right)
$$

where $\Delta_{a b}^{*}$ is the value that defines a thermally activated process across the energy gap - the pseudogap. The exponential dependence of $\Delta \sigma(T)$ was also observed earlier in YBCO film samples [3]. Prokofyev et al. [3] demonstrated that the description of experimental data can be significantly improved by introducing the factor $\left(1-T / T^{*}\right)$. The excess conductivity becomes proportional to the superconducting carrier den- 
sity $n_{s} \sim\left(1-T / T^{*}\right)$ and inversely proportional to the number of pairs $\sim \exp \left(\Delta_{a b}^{*} / T\right)$, that are destroyed by thermal motion

$$
\Delta \sigma \sim\left(1-T / T^{*}\right) \exp \left(\Delta_{a b}^{*} / T\right) .
$$

Thus $T^{*}$ is the temperature of superconducting transition in the mean-field approximation. The temperature interval $T_{\mathrm{c}}<T<T^{*}$, in which the $\mathrm{PG}$ regime exists is defined by the order parameter of the specific phase, which depends on either the oxygen nonstoichiometry or the dopant concentration. Therefore, using the method proposed by Prokofyev et al. [3], we can plot the experimental curve $\ln \Delta \sigma$ for the temperature dependence $\Delta_{a b}^{*}(T)$ for temperatures lower than $T^{*}$. Figure 2 shows the temperature dependence of the PG i.e. $\Delta^{*}(T) / \Delta_{\max }$ versus $T / T^{*}$, where $\Delta_{\max }$ is the $\Delta_{a b}^{*}$ value in the plateau, away from $T^{*}$, measured under different pressures.

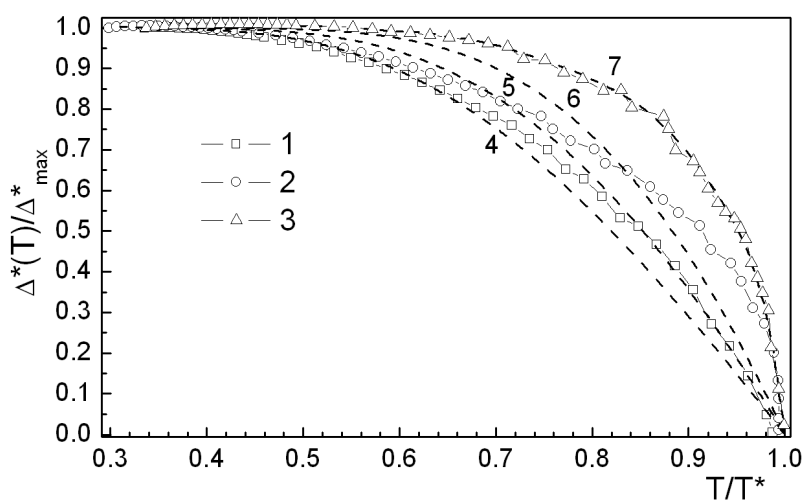

Fig. 2. Temperature dependence of the pseudogap of $\mathrm{HoBa}_{2} \mathrm{Cu}_{3} \mathrm{O}_{7-\delta}$ single crystal in $\Delta^{*}(T) / \Delta_{\max }^{*}$ versus $T / T^{*}$ coordinates $\left(\Delta_{\max }^{*}\right.$ is the value of $\Delta^{*}$ in the plateau, away from $\left.T^{*}\right)$. The numbering of the curves is in accordance to Fig. 1. The dashed lines show the $\Delta^{*}(T) / \Delta(0)$ versus $T / T^{*}$ dependence, calculated according to Eq. (7), for the crossover parameter $\mu / \Delta(0)=10$ (limit of the BCS theory) and $\mu / \Delta(0)=$ $-2,-5,-10$ (limit of the BEC theory), curves $4-7$, respectively.

The PG temperature dependence was described in [7] using the crossover mechanism from the BCS-BEC theory assuming the weak as well as strong coupling. According to this theory the temperature dependence of PG is given by the following formula:

$$
\begin{aligned}
& \Delta(T)=\Delta(0)-\Delta(0) \sqrt{\frac{\pi}{2}} \sqrt{\frac{T}{\Delta(0)}} \exp \left(\frac{\Delta(0)}{T}\right) \\
& \times\left[1+\operatorname{erf}\left(\sqrt{\frac{\sqrt{x_{0}^{2}+1}-1}{T / \Delta(0)}}\right)\right],
\end{aligned}
$$

where $x_{0}=\mu / \Delta(0), \mu$ is the chemical potential of the carrier system and $\Delta(0)$ is the value of energy gap at the zero temperature and $\operatorname{erf}(x)$ is the error function.
When $x_{0} \rightarrow \infty$ (weak coupling), Eq. (4) becomes

$$
\Delta(T)=\Delta(0)-\Delta(0) \sqrt{2 \pi \Delta(0) T} \exp \left(\frac{\Delta(0)}{T}\right),
$$

which is a well-known result in the BCS theory. In the limit of strong interactions in the three-dimensional case $\left(x_{0}<-1\right)$, Eq. (4) becomes

$$
\begin{aligned}
& \Delta(T)=\Delta(0)-\frac{8}{\sqrt{\pi}} \sqrt{-x_{0}}\left(\frac{\Delta(0)}{T}\right)^{3 / 2} \\
& \times \exp \left(-\frac{\sqrt{\mu^{2}+\Delta^{2}(0)}}{T}\right) .
\end{aligned}
$$

The $\Delta^{*}(T) / \Delta(0)$ versus $T / T^{*}$ dependence is calculated using Eqs. (5), (6) in the mean-field approximation within the context of the BCS-BEC crossover theory [7]. The values of the crossover parameters $\mu / \Delta(0)=10$ (limit of BCS) and $\mu / \Delta(0)=-2,-5,-10$ (limit of BEC) are shown in Fig. 2 as the dotted lines. This figure indicates that the increase in the pressure applied results in a deviation of the experimental curves from those described by Eq. (6) (strong coupling) to Eq. (5) (weak coupling). This behaviour is qualitatively similar to the effect of transformation of pseudogap temperature dependence observed for YBCO samples under application of high pressure [8]. It is obvious that these correlations in the behaviour of the curves $\Delta^{*}(T)$ are not accidental. Indeed, as well known from literature (see e.g. [6, 8]), the application of high pressure to the samples of HTSC 1-2-3 system, as well as an increase in the oxygen content lead to improved conductive properties, which include an increase in the critical temperature and a significant reduction of the electrical resistivity. Consequently, assuming the value of $T^{*}$ as a temperature at which pseudogap commences (i.e. at which the deviation of $\Delta(T)$ from the linear behaviour appears) the experiment is consistent with theory.

The inset (b) of Fig. 1 shows the time dependence of temperature $T^{*}$ upon the gradual annealing of the samples to room temperature $T^{*}(t)$. The solid lines correspond to the fit with the expression proposed by Jorgensen et al. [9]:

$$
T^{*}(t)=T^{*}(\infty)+\left[T^{*}(0)-T^{*}(\infty)\right] \exp \left(-(t / \tau)^{1 / 2}\right),
$$

where $T^{*}(\infty)$ and $T^{*}(0)$ are the equilibrium and the initial value of $T^{*}$, respectively, and $\tau$ is a characteristic time of the relaxation process. As follows from the calculations with the use of (7), the equilibrium value of $T^{*}(\infty)$ in the process of gradual annealing is reached within $9.5 \mathrm{~h}$. Using the values of $\tau$, obtained by Eq. (7) using our experimental data, we can determine the activation energy of the relaxation process in our samples [6] using the Arrhenius law

$$
\tau=\tau_{0} \exp \left(\frac{E_{\mathrm{A}}}{k_{\mathrm{B}} T}\right),
$$


where $\tau_{0}=1.4 \times 10^{-12} \mathrm{~s}$ is the characteristic time [10], which, according to [6], is independent of pressure. The activation energy obtained from our data $E_{\mathrm{A}} \approx$ $0.94 \mathrm{eV}$ is slightly lower than the typical values for the $\mathrm{YBa}_{2} \mathrm{Cu}_{3} \mathrm{O}_{7-\delta}$ compounds with reduced oxygen concentration [11]. It should also be noted that all the characteristic shape changes in the temperature dependence and absolute values of the resistivity parameters that were observed in the isobaric annealing process at room temperature were more pronounced in $\mathrm{HoBa}_{2} \mathrm{Cu}_{3} \mathrm{O}_{7-\delta}$ than in $\mathrm{YBa}_{2} \mathrm{Cu}_{3} \mathrm{O}_{7-\delta}$. In $\mathrm{HoBa}_{2} \mathrm{Cu}_{3} \mathrm{O}_{7-\delta}$ the Ho ion (which has larger ionic radius than $\mathrm{Y}$ ) plays an important role in the structural ordering of the system, which affects the interactions between oxygen ions in the $\mathrm{CuO}$ planes. Earlier study revealed that when $\mathrm{Y}$ is substituted by the rare-earth elements with larger ionic radius, significant qualitative changes in the $T_{\mathrm{c}}(\delta)$ dependence in $\mathrm{ReBa}_{2} \mathrm{Cu}_{3} \mathrm{O}_{7-\delta}[12]$ appear. The $T_{\mathrm{c}}(\delta)$ dependence characteristic of $\mathrm{YBa}_{2} \mathrm{Cu}_{3} \mathrm{O}_{7-\delta}$ with two plateaus at $60 \mathrm{~K}$ and $90 \mathrm{~K}$ degenerates into a usual monotonically dependence. Additionally, the ortho-II structure is not realized at all [12]. It can be assumed that oxygen hypostoichiometric $\mathrm{HoBa}_{2} \mathrm{Cu}_{3} \mathrm{O}_{7-\delta}$ compounds have a more extended disordered oxygen hyperstructure than $\mathrm{YBa}_{2} \mathrm{Cu}_{3} \mathrm{O}_{7-\delta}$ compounds.

\section{Conclusions}

The redistribution of the labile oxygen induced by annealing of $\mathrm{HoBa}_{2} \mathrm{Cu}_{3} \mathrm{O}_{7-\delta}$ crystals results in a significant expansion of the interval of the linear relationship $\rho_{a b}(T)$ and narrowing of the temperature range of the pseudogap regime. The excess conductivity is manifested as exponential temperature dependence in a wide temperature range. The temperature dependence of the pseudogap is well described in the framework of the BCS-BEC crossover theory.

\section{References}

[1] R.V. Vovk, M.A. Obolenskiy, A.A. Zavgorodniy, D.A. Lotnyk, K.A. Kotvitskaya, Physica B 404, 3516 (2009).

[2] M. Sadovskii, Phys. Usp. 171, 539 (2001).

[3] D.D. Prokofyev, M.P. Volkov, Yu.A. Boikov, Fiz. Tverd. Tela 45, 1168 (2003) [Phys. Solid State 45, 1223 (2003).

[4] R.V. Vovk, M.A. Obolenskii, A.V. Bondarenko, I.L. Goulatis, A.V. Samoilov, A. Chroneos, V.M. Pinto Simoes, J. Alloys Comp. 464, 58 (2008).

[5] R.V. Vovk, M.A. Obolenskii, A.A. Zavgorodniy, I.L. Goulatis, V.I. Beletskii, A. Chroneos, Physica $C$ 469, 203 (2009).

[6] S. Sadewasser, J.S. Schilling, A.P. Paulicas, B.M. Veal, Phys. Rev. B 61, 741 (2000).

[7] E. Babaev, H. Kleinert, Phys. Rev. B 59, 12083 (1999).

[8] R.V. Vovk, A.A. Zavgorodniy, M.A. Obolenskii, I.L. Goulatis, A. Chroneos, V.M. Pinto Simoes, Mod. Phys. Lett. B 24, 2295 (2010).

[9] D. Jorgensen, P. Shiyou, P. Lightfoot, H. Shi, A.P. Paulikas, B.M.W. Veal, Physica C 167, 571 (1990).

[10] B.W. Veal, H. You, A.P. Paulicas, H. Shi, Y. Fang, J.W. Downey, Phys. Rev. B. 42, 4770 (1990).

[11] A. Chroneos, R.V. Vovk, I.L. Goulatis, L.I. Goulatis, J. Alloys Comp. 494, 190 (2010).

[12] H. Lutgemeier, S. Schmenn, P. Meuffels, O. Storz, R. Schollhorn, Ch. Niedermayer, I. Heinmaa, Yu. Baikov, Physica C 267, 191 (1996). 